\title{
Quantifying Institutional Reach Through the Human Network in Natural History Collections
}

\author{
David Peter Shorthouse ${ }^{\ddagger}$, Roderic Page ${ }^{\S}$ \\ ‡ Agriculture \& Agri-Food Canada, Ottawa, Canada \\ $\S$ University of Glasgow, Glasgow, United Kingdom
}

Corresponding author: David Peter Shorthouse (davidpshorthouse@gmail.com)

Received: 07 Apr 2019 | Published: 08 Aug 2019

Citation: Shorthouse DP, Page R (2019) Quantifying Institutional Reach Through the Human Network in Natural

History Collections. Biodiversity Information Science and Standards 3: e35243. https://doi.org/10.3897/biss.3.35243

\begin{abstract}
Through the Bloodhound proof-of-concept, https://bloodhound-tracker.net an international audience of collectors and determiners of natural history specimens are engaged in the emotive act of claiming their specimens and attributing other specimens to living and deceased mentors and colleagues. Behind the scenes, these claims build links between Open Researcher and Contributor Identifiers (ORCID, https://orcid.org) or Wikidata identifiers for people and Global Biodiversity Information Facility (GBIF) specimen identifiers, predicated by the Darwin Core terms, recordedBy (collected) and identifiedBy (determined). Here we additionally describe the socio-technical challenge in unequivocally resolving people names in legacy specimen data and propose lightweight and reusable solutions. The unique identifiers for the affiliations of active researchers are obtained from ORCID whereas the unique identifiers for institutions where specimens are actively curated are resolved through Wikidata. By constructing closed loops of links between person, specimen, and institution, an interesting suite of potential metrics emerges, all due to the activities of employees and their network of professional relationships. This approach balances a desire for individuals to receive formal recognition for their efforts in natural history collections with that of an institutional-level need to alter budgets in response to easily obtained numeric trends in national and international reach. If handled in a coordinating fashion, this reporting technique may be a significant new driver for specimen digitization efforts on par with Altmetric, https://www.altmetric.com, an important new tool that tracks the impact of publications and delights administrators and authors alike.
\end{abstract}




\section{Keywords}

ORCID, wikidata, specimens, people, institutions, credit systems

\section{Presenting author}

David Shorthouse 